\title{
Capital social no Brasil: uma análise de seus determinantes ${ }^{1}$
}

\author{
Social capital in Brazil: an analysis of its determinants
}

\author{
Capital social en Brasil: un análisis de sus determinantes
}

\author{
Lilian Lopes Ribeiro ${ }^{2}$ \\ Lucas Tomaz Boto \\ Fernando Daniel de Oliveira Mayorga²
}

Recebido em 08/03/2020; revisado e aprovado em 10/08/2020; aceito em 21/08/2020. DOI: http://dx.doi.org/10.20435/inter.v21i4.2959

Resumo: O artigo analisa os fatores determinantes do capital social no Brasil. Com o intuito de atender esse objetivo, calculou-se inicialmente um índice de capital social com base na técnica dos componentes principais. São considerados para construção desse índice um total de vinte indicadores extraídos da World Values Survey (WVS), onze deles relacionados à participação do entrevistado em organizações/associações e nove referentes à confiança interpessoal. A análise é feita a partir dos efeitos marginais do modelo logit. Entre os resultados obtidos, constatou-se que a variável que mais tende a contribuir para a formação de capital social é a educação. Ter um curso superior tende a aumentar as chances de um indivíduo ter, por exemplo, uma dotação "muito alta" de capital social em 14\%. Observou-se também que quem mora nas regiões Centro-Oeste e Sul do Brasil é mais propenso a possuir um nível mais elevado desse capital. Já as variáveis dummies relacionadas ao gênero e à raça não apresentaram significância do ponto de vista estatístico. Deste modo, o fator discriminação, tendo como base essas variáveis, parece não interferir no processo de formação de capital social dos brasileiros. Como proposição de política, recomenda-se a intensificação de políticas públicas direcionadas fundamentalmente à expansão e à qualidade da educação no Brasil, uma vez que esse é o principal fator contribuinte para a formação de um alto nível de capital social no país.

Palavras-chave: capital social; coesões sociais; World Values Survey.

Abstract: The article analyzes the determinants of social capital in Brazil. To meet this objective, we initially calculated a social capital index based on the principal component technique. For the construction of this index, we considered twenty indicators taken from the World Values Survey (WVS), eleven of them related to the interviewed participation in organizations/associations, and nine referring to interpersonal trust. The analysis is based on the marginal effects of the logit model. Among the results obtained, we found that the variable that most tends to contribute to the formation of social capital is education. Having a college degree tends to increase the chances of an individual having, for example, a "very high" endowment of social capital by $14 \%$. We also observed that those who live in the Midwest and South regions of Brazil are more likely to have a higher level of this capital. The dummies variables related to gender and race were not statistically significant. Thus, the discrimination factor, based on these variables, does not seem to interfere in the process of forming the social capital of Brazilians. As a policy proposition, we recommended to intensify public policies aimed primarily at the expansion and quality of education in Brazil, since this is the main contributing factor for the formation of a high level of social capital in the country.

Keywords: social capital; social cohesion; World Values Survey.

Resumen: El artículo analiza los determinantes del capital social en Brasil. Para alcanzar este objetivo, se calculó inicialmente un índice de capital social basado en la técnica del componente principal. Para la construcción de este índice, se consideran un total de veinte indicadores tomados de la Encuesta Mundial de Valores (WVS), once de ellos relacionados con la participación del entrevistado en organizaciones/asociaciones y nueve referidos a la confianza interpersonal. El análisis se basa en los efectos marginales del modelo logit. Entre los resultados obtenidos, se encontró que la variable que más tiende a contribuir a la formación de capital social es la educación. Tener un título universitario tiende a aumentar las posibilidades de que un individuo tenga, por ejemplo, una dotación de capital social "muy alta" en un 14\%. También se observó que aquellos que viven en las regiones del Medio Oeste y Sur de Brasil tienen más probabilidades de tener un mayor nivel de esta capital. Las variables dummies relacionadas con el género y la raza no fueron estadísticamente significativas. Por lo tanto, el factor de discriminación, basado en estas variables, no parece interferir en el proceso de formación del capital social de los brasileños. Como propuesta de política, se recomienda

\footnotetext{
${ }^{1}$ Este trabalho foi financiado pela Fundação Cearense de Apoio ao Desenvolvimento Científico e Tecnológico (Funcap).

${ }^{2}$ Universidade Federal do Ceará (UFC), Sobral, Ceará, Brasil.
} 
intensificar las políticas públicas dirigidas principalmente a la expansión y la calidad de la educación en Brasil, ya que este es el principal factor que contribuye a la formación de un alto nivel de capital social en el país.

Palabras claves: capital social; cohesión social; Encuesta Mundial de Valores.

\section{INTRODUÇÃO}

Em linhas gerais, o capital social cívico pode ser definido como resultante de coesões ou redes sociais, formais ou informais, com objetivos comuns que são pautados em normas e que compartilham alguns atributos, como: cooperação mútua; confiança interpessoal; solidariedade; reciprocidade e tolerância (Tenzin; OTSUKA; NATSUDA, 2013).

Mas, afinal, por que estudar os determinantes do capital social? A resposta a essa pergunta pode ser encontrada em diversos estudos acerca do tema, como o de Johannes (2009), que revela que essa modalidade de capital pode contribuir positivamente para o desenvolvimento econômico. Não obstante, uma importante referência de estudo empírico que comprova tal assertiva é Putnam et al. (1993).

Ao analisarem a participação cívica e o desempenho institucional entre as regiões Norte e Sul da Itália ao longo de vinte anos, Putnam et al. (1994) observaram que as regiões que obtiveram um melhor desempenho institucional e cívico não coincidiram com aquelas que receberam mais recursos financeiros, e sim com aquelas que praticaram, entre outros fatores, laços fortes de confiança mútua, de solidariedade interpessoal e de democracia participativa.

O trabalho seminal de Putnam et al. (1994) inspirou diversos outros pesquisadores a estudar as benesses que o capital social pode trazer para a economia e que, em grande medida, estão conectadas com o desenvolvimento econômico de um país ou região.

Em síntese, esses estudos mostraram que o capital social pode diminuir a desigualdade de renda (KNACK, 2002); reduzir os riscos associados à insegurança alimentar (MARTIN et al, 2004); reduzir as falhas de mercado e comportamentos free-rider (ANDRIANI; KARYAMPAS, 2015); facilitar a microcooperativa de negócios, gerando renda aos seus participantes (PEÑALOZA, 2013); induzir a um controle eficiente da prestação de serviços do governo (EVANS, 1996; HASSAN; BIRUNGI, 2011); e também aumentar a produtividade na economia (KNACK; KEEFER, 1997). Outras pesquisas evidenciam a contribuição desse capital na redução da pobreza. Entre essas, é oportuno destacar as mais atuais: Ribeiro e Araújo (2018); Tenzin; Otsuka e Natsuda (2013) e ljaiya et al. (2012).

Diante de tantas evidências apontando para o quão relevante pode ser o capital social para o desenvolvimento da economia, torna-se de fundamental importância conhecer e analisar os possíveis fatores capazes de determinar esse capital. Certamente, este estudo pode corroborar para uma melhor aplicabilidade de políticas públicas. Vale salientar também que, no Brasil, praticamente não há estudos econômicos para tal análise. Essas são, portanto, importantes motivações para a realização deste estudo.

Assim sendo, o objetivo deste estudo é analisar os determinantes do capital social no Brasil, por meio de um índice de capital social construído com base na técnica de análise multivariada denominada de componentes principais e a partir de indicadores de confiança e de participação dos entrevistados em organizações/associações disponibilizados pela World Values Survey (WVS). Estimaram-se os resultados por meio do modelo logit, seguido de seus efeitos marginais.

Além da introdução, o artigo apresenta mais quatro seções. A segunda apresenta uma breve revisão da literatura sobre o capital social e seus determinantes. Na terceira seção, encontram- 
se os dados amostrais, a apresentação das variáveis utilizadas na pesquisa e a descrição da construção do índice de capital social, além da formalização do modelo econométrico utilizado. A quarta, por sua vez, apresenta os resultados produzidos e suas análises. E a última é dedicada às considerações finais do artigo.

\section{REFERENCIAL TEÓRICO}

\subsection{Uma breve revisão do conceito}

São poucas as teorias nas ciências sociais aplicadas que surgiram e foram ampla e rapidamente utilizadas no processo investigativo das relações sociais. Sem sombra de dúvidas, o conceito de capital social é uma delas. Apesar da noção de associação entre indivíduos em comunidade ter uma longa história, o uso do conceito de capital social nas ciências humanas tem sua origem no pós-guerra, e sua importância é reconhecida nas décadas de 1970 e 1980, com Bourdieu (1986), Wacquant (BOURDIEU; WACQUANT, 1992) e Coleman (1988).

Putnam expandiu o conceito na década de 1990, visando a uma contribuição nas ciências sociais $(1993,1995)$, e em 2000, visando ao público em geral, escreveu o livro Bowling Alone: The Collapse and Revival of American Community, em que verificou grande variação dos fatores que influenciam a criação e o fortalecimento do capital social nos Estados Unidos.

Uma das características marcantes do conceito de capital social é a grande quantidade de definições que lhe são atribuídas com o objetivo de explicar as relações cívicas e institucionais na sociedade. Porém, em síntese, o conceito pode ser dividido em dois grupos, ou duas perspectivas. Uma vê o capital social como uma fonte de recurso (capital) criado por meio das ações racionais dos indivíduos (BOURDIEU; WACQUANT, 1992; PALDAM, 2000; BROOKS, 2005), e a outra é a perspectiva de imersão ('embeddedness'), que foca nos resultados e na criação do capital social por meio da coletividade (COLEMAN, 1988; PUTNAM, 1995; BEBBINGTON; PERREAULT, 2008).

O presente estudo, ao analisar os determinantes do capital social, procura contemplar as duas perspectivas por meio de indicadores em nível individual, como educação formal, e indicadores gerais, como engajamento social e participação cívica.

\subsection{Determinantes do capital social}

Dada a abrangência do conceito, a medição do capital social tem se mostrado um desafio aos estudiosos (INKELES, 2000). Uma das primeiras investidas realizadas foi de Lochner; Kawachi e Kennedy (1999), cujo objetivo foi de estabelecer um guia para operacionalizar e medir capital social, por meio de indicadores em nível de indivíduo, assim como em nível coletivo. A conclusão foi de que tem de se levar em consideração o nível em que está sendo medido o capital social, se em nível de comunidade ou em nível de município ou estado. Ou seja, o nível de análise vai influenciar nas variáveis a serem consideradas, no método a ser utilizado e, consequentemente nos resultados obtidos.

Já Onyx e Bullen (2000) utilizaram análise fatorial para identificar os principais determinantes do capital social em cinco comunidades australianas. Os autores concluíram que os três fatores mais significantes foram: participação ativa em organizações cívicas e eventos locais; comportamento proativo em um contexto social; e, por último, sentimentos de confiança e segurança na comunidade em que residem. Esses três fatores foram responsáveis por mais de 
30\% da variância, sendo elementos centrais do conceito de capital social discutido na literatura como participação em redes sociais, confiança e proatividade social.

Narayan e Cassidy (2001) foram os primeiros a elaborarem um questionário validado para medir os determinantes do capital social. Um dos objetivos do questionário foi o de diferenciar, entre os determinantes, as dimensões e os resultados ou benefícios advindos do capital social. Sendo o questionário aplicado na República do Gana e, posteriormente, em Uganda, os autores concluíram que o engajamento político, empoderamento social e solidariedade em nível comunitário são fatores fundamentais na criação do capital social.

Christoforou (2005) faz uma comparação dos determinantes do capital social entre a Grécia e os demais países da União Europeia e como esse capital influencia no crescimento econômico, incentivando instituições e comportamentos individuais que aumentam a produtividade. $O$ autor conclui que os determinantes variam em razão das diferentes formas de regulação social e suas interações com as condições socioeconômicas apresentadas, assim como as influências institucionais que são específicas à cultura e história de cada nação.

Além disso, Christoforou (2005) constatou que o nível educacional e estar empregado aumentam as chances de socialização e construção de confiança entre os indivíduos, fundamentais para criação de capital social. Em termos agregados, o autor observa que a baixa renda per capita e altos índices de desigualdade são desestímulos para a formação de coesões sociais.

Alexander (2007), por sua vez, faz uma comparação do nível de capital social nos Estados Unidos, utilizando como base os resultados encontrados por Putnam (2000). Os resultados apontam que existe um declínio no nível de capital social observado, não somente no tempo, mas também no espaço. Indicadores socioeconômicos, como educação, participação em atividades religiosas e desemprego, são os principais fatores que explicam em grande parte a variação espaço-temporal do capital social.

Downing (2011), tendo como base os dados da SCBS (Social Capital Benchmark Survey), ao analisar diversos fatores determinantes na criação do capital social, chega à conclusão de que a educação é a variável-chave e que existe uma relação forte entre o nível educacional de uma sociedade e seu nível de capital social. Outro fator importante citado pelo autor quanto ao nível educacional é que este tende a influenciar políticas públicas que incentivam a interação social entre os indivíduos de uma sociedade, criando um círculo vicioso benéfico à sociedade.

Por fim, Parts (2013), que analisou as dinâmicas e os determinantes do capital social nos países da União Europeia, identificou quatro fatores fundamentais, sendo eles: a confiança geral; confiança institucional; interação social; e normas sociais. Seu estudo conclui que o nível educacional e a satisfação com as instituições democráticas têm relação positiva com o nível de capital social, sendo prudente, portanto, investimentos com objetivos de melhorias no sistema educacional e processos democráticos.

\section{METODOLOGIA}

Esta seção é dedicada a uma breve descrição dos dados utilizados neste trabalho, aos detalhes do cálculo dos componentes principais e à apresentação e especificação do modelo econométrico usado para analisar os condicionates do capital social no Brasil. 


\subsection{DADOS AMOSTRAIS}

Para explicar os condicionantes do capital social no Brasil, foi utilizado um conjunto de variáveis extraídas do banco de dados da Word Values Survey (WVS) ${ }^{3}$, que resultou em 1.118 observações e que pode ser desagregado em duas partes. O primeiro conjunto, apresentado na Tabela 1 , diz respeito às variáveis utilizadas no cálculo do índice de capital social. São variáveis resultantes de perguntas destinadas ao entrevistado sobre sua participação em organizações ou associações e a confiança que atribui às pessoas.

Tabela 1 - Especificação das variáveis para a construção do índice de capital social

\begin{tabular}{c|c|c}
\hline Categoria & Variável & Descrição \\
\hline Dicotômica & V24 & Se pode confiar na maioria das pessoas ou se é preciso ser muito \\
cuidadoso.
\end{tabular}

Fonte: WVS. Elaboração do autor.

\footnotetext{
${ }^{3}$ A WVS é uma organização não governamental, existente desde 1981, que explora valores e crenças das pessoas, aplicando pesquisas em diversos países no mundo acerca da democracia; tolerância; bem-estar subjetivo; política; religião; entre outros temas. No Brasil, a pesquisa foi realizada nos anos de 1991, 2006 e 2014.
} 
O critério de escolha das variáveis para compor o índice de capital social pode ser vislumbrado em Putnam et al. (1994) e em vários artigos científicos sobre o tema, como o trabalho de Hassan e Birungi (2011). Além disso, tais variáveis são inerentes do próprio conceito de capital social apresentado na seção anterior.

Já o segundo conjunto, detalhado na Tabela 2, é constituído pelas variáveis explicativas usadas na estimação do modelo econométrico e relacionadas às características pessoais dos entrevistados. São elas: escala de renda e idade e um total de quinze dummies construídas a partir das categorias de variáveis sexo (ou gênero); nível educacional mais alto; grupo étnico; religião e região.

Tabela 2- Descrição das variáveis explicativas

\begin{tabular}{c|c|l}
\hline Categoria & Variável & \multicolumn{1}{c}{ Descrição } \\
\hline Escalar & EscRenda & $\begin{array}{l}\text { Escala de rendimentos, sendo de 1 (mais } \\
\text { baixo) a 10 (mais alto) }\end{array}$ \\
Numérica & Idade & Idade \\
Dicotômica & Ida2 & Idade ao quadrado \\
Dicotômica & DMasc & Sexo masculino \\
Dicotômica & DSup & Ensino superior completo \\
Dicotômica & DAnalf & Sem escolaridade ou primário incompleto \\
Dicotômica & DOutrEsC & Outras escolaridades \\
Dicotômica & DBran & Branco \\
Dicotômica & DNegr & Negro \\
Dicotômica & DOutrRacas & Outras raças (indígenas, asiáticos etc.) \\
Dicotômica & DCatol & Católico \\
Dicotômica & DEvang & Evangélico ou protestante \\
Dicotômica & DOutrRelig & Outras religiões ou sem religião \\
Dicotômica & DNO & Reside no Norte \\
Dicotômica & DNE & Reside no Nordeste \\
Dicotômica & DCO & Reside no Centro-Oeste \\
Dicotômica & DSE & Reside no Sudeste \\
\hline
\end{tabular}

Fonte: WVS. Elaboração do autor.

A inclusão das variáveis dummies DMasc, DBran, DNegr e DOutrEsc tem como finalidade examinar se a discriminação de gênero e de raça interfere no processo de formação de capital social. Sobre a inclusão especificamente das dummies relacionadas à religião, essas têm por finalidade comprovar ou refutar a assertiva de Putnam et al. (1994) de que, entre os católicos, pode haver um baixo estoque de capital social, comparativamente aos protestantes, devido ao fato de que as relações interpessoais entre esse grupo de religiosos tendem a ser mais verticais que horizontais, prejudicando, assim, a formação de capital social.

\subsection{Cálculo do índice de capital social}

Para a construção do índice agregado de capital social, tendo como base as variáveis descritas na Tabela 1, utilizou-se uma técnica de análise multivariada denominada de componentes principais. O objetivo dessa técnica é obter pesos que traduzam a importância 
das variáveis, obtendo, assim, combinações lineares das variáveis. Essas combinações lineares são chamadas de componentes principais e são não correlacionadas entre si. Tais combinações possibilitam capturar o máximo de variância possível, tendo como base a decomposição da matriz de covariâncias (MINGOTI, 2007). Em síntese, conforme relata Regazzi (2000), a técnica de componentes principais pode ser associada à ideia de redução de massa de dados, com menor perda possível da informação.

Considere então $X=\left(X_{1}, X_{2} \ldots X_{j}\right)^{\prime}$ como um vetor aleatório de variáveis com vetor de médias $\mu=\left(\mu_{1}, \mu_{2} \ldots \mu_{j}\right)$ e matriz de covariâncias $\Sigma_{p x p}$. Sejam $\lambda_{1} \geq \lambda_{2} \geq \ldots \geq \lambda_{p}$ os autovalores da matriz $\Sigma_{p x p}$ com os respectivos autovetores denotados por $e_{i}=\left(e_{i 1}, e_{i 2}, \ldots e_{i j}\right)^{\prime}$. Nesses termos, a n-ésima componente principal para uma observação $j$ da amostra pode ser expressa por $C_{n}^{j}=\sum_{j} e_{i j} X_{j}{ }^{4}$. Logo, para cada uma das 1.118 observações deste estudo, obteve-se um Índice de Capital Social dado por $I C S_{j}=\frac{\sum_{n} \lambda_{n} c_{n}^{j}}{\sum_{n=1} \lambda_{n}}$.

Ressalta-se também que a variância acumulada dos componentes principais deve representar uma porcentagem acima de 80\% (MANLY, 2008). Deste modo, para o cálculo do ICS, foram utilizados os autovalores dos quinze primeiros componentes principais gerados. Esses componentes, por sua vez, explicam 88,54\% da variância total das variáveis da amostra.

Para que fosse factivel uma análise preliminar de identificação dos níveis de estoque de capital social das regiões brasileiras - subseção 4.1 -, quatro níveis foram estabelecidos a partir dos valores encontrados para o ICS:

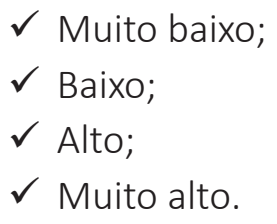

A variável ordenada "níveis de capital social" assumiu então os seguintes valores: 1 , quando a categoria é "muito baixo"; 2, quando a categoria é "baixo"; 3, quando a categoria é "alto"; e 4, quando a categoria é "Muito alto". Desse modo, o modelo apropriado para a estimação dos resultados é o Logit Ordenado, uma vez que se tem uma variável dependente na forma discreta e latente. Esse modelo está especificado na subseção a seguir.

\subsection{Modelo econométrico}

O modelo logit ordenado é empregado para estimar valores de variáveis latentes por meio de equações lineares. Esse modelo pode ser vislumbrado como um modelo de regressão linear para uma variável resposta contínua não observada (latente) na forma:

$$
y_{i}^{*}=X_{i} \beta_{i}+\varepsilon_{i} \quad i=1, \ldots \ldots, k .
$$

em que $y_{i}$ é uma variável discreta e latente, sendo $X_{i}$ o vetor de variáveis explicativas descritas na Subseção 3.1 e $\boldsymbol{\beta}_{i}$ o coeficiente de $X_{i}$. O erro aleatório, $\boldsymbol{\varepsilon}_{i}$, segue uma distribuição logística por hipótese. Sendo o subescrito $i$ relativo ao indivíduo na amostra de tamanho k.

\footnotetext{
${ }^{4}$ Para $j$ - variáveis originais, é possível obter $j$ componentes principais.
} 
Como o modelo logit, ao contrário do método dos Mínimos Quadrados Ordinários, não possibilita a interpretação dos estimadores diretamente da regressão, para a análise a ser feita é necessário o cálculo de seus efeitos marginais. Nesses termos, os efeitos marginais do modelo logit ordenado podem ser denotados por:

$$
\frac{\partial \operatorname{Pr}\left[y_{i}=j^{\prime} / X\right]}{\partial x_{k, i}}=\left(\frac{\partial F\left(\alpha_{j}-X_{i} \beta^{\prime}\right.}{\partial x_{k, i}}-\frac{\partial F\left(\alpha_{j-1}-X_{i} \beta^{\prime}\right.}{\partial x_{k, i}}\right)=\beta_{k}\left(f\left(\alpha_{j-1}-X_{i} \beta^{\prime}\right)-f\left(\alpha_{j}-X_{i} \beta^{\prime}\right)\right.
$$

sendo que os efeitos marginais não dependem apenas do coeficiente $\boldsymbol{\beta}_{\boldsymbol{k}}$, mas também da função densidade $f($.$) .$

O uso do logit ordenado se justifica devido ao fato de esse modelo possuir a propriedade de explicar variáveis categóricas qualitativas e discretas que apresentam um número maior de alternativas, quando comparado com as variáveis dicotômicas (Powers; Xie, 2000). Tais características apresentadas pelo logit se enquadram no perfil da equação estimada nesta pesquisa. Ressalta-se também o fato de que, como a distribuição logística tem uma forma analítica fechada, o custo - em termos computacionais - de estimá-lo tende a ser menor comparado ao do modelo probit, uma vez que a distribuição normal desse modelo é uma integral que precisa ser computada numericamente.

\section{ANÁLISE DOS RESULTADOS}

Esta seção está subdividida em duas partes. Na primeira parte, são apresentados os níveis de capital social para o Brasil e para as cinco regiões brasileiras, sendo a segunda dedicada aos resultados e à análise do modelo logit ordenado e seus efeitos marginais.

\subsection{Estoques de capital social das regiões brasileiras}

O Mapa 1 apresenta, em termos percentuais, os níveis dos estoques de capital social para as regiões brasileiras para o ano de 2014.

Pelo mapa, é possível observar que a região Norte é, entre as cinco regiões, aquela que mais apresenta ocorrências de piores níveis de capital social. Cerca de 32\% do capital social existente nessa região pode ser classificado como sendo "muito baixo". A região Sudeste vem logo em seguida, com $27,56 \%$ de seu capital social classificado nessa categoria. A soma dos percentuais de capital social classificados como "muito baixo" e "baixo" para o Norte e para o Sudeste brasileiro totaliza um valor de 53,53\% e 52,67\%, respectivamente, evidenciando um baixo estoque de capital social para as duas regiões. Esses resultados evidenciam, de modo intrínseco, não somente um baixo engajamento cívico da sociedade como também um baixo nível de confiança entre as pessoas, sobretudo no Norte do país.

Em outra direção, nas regiões Centro-Oeste, Sul e Nordeste, há uma predominância de alto estoque de capital social, já que há uma maior ocorrência de capital social classificado como "alto" e "muito alto" nessas três regiões. No caso do Centro-Oeste, cerca de 33\% do capital social pode ser classificado, por exemplo, como sendo "muito alto"; no Sul do Brasil, esse percentual é de 30,68\%; e no Nordeste, de 21,78\%. 
Mapa 1 - Distribuição dos níveis de capital social por região brasileira (em \%)

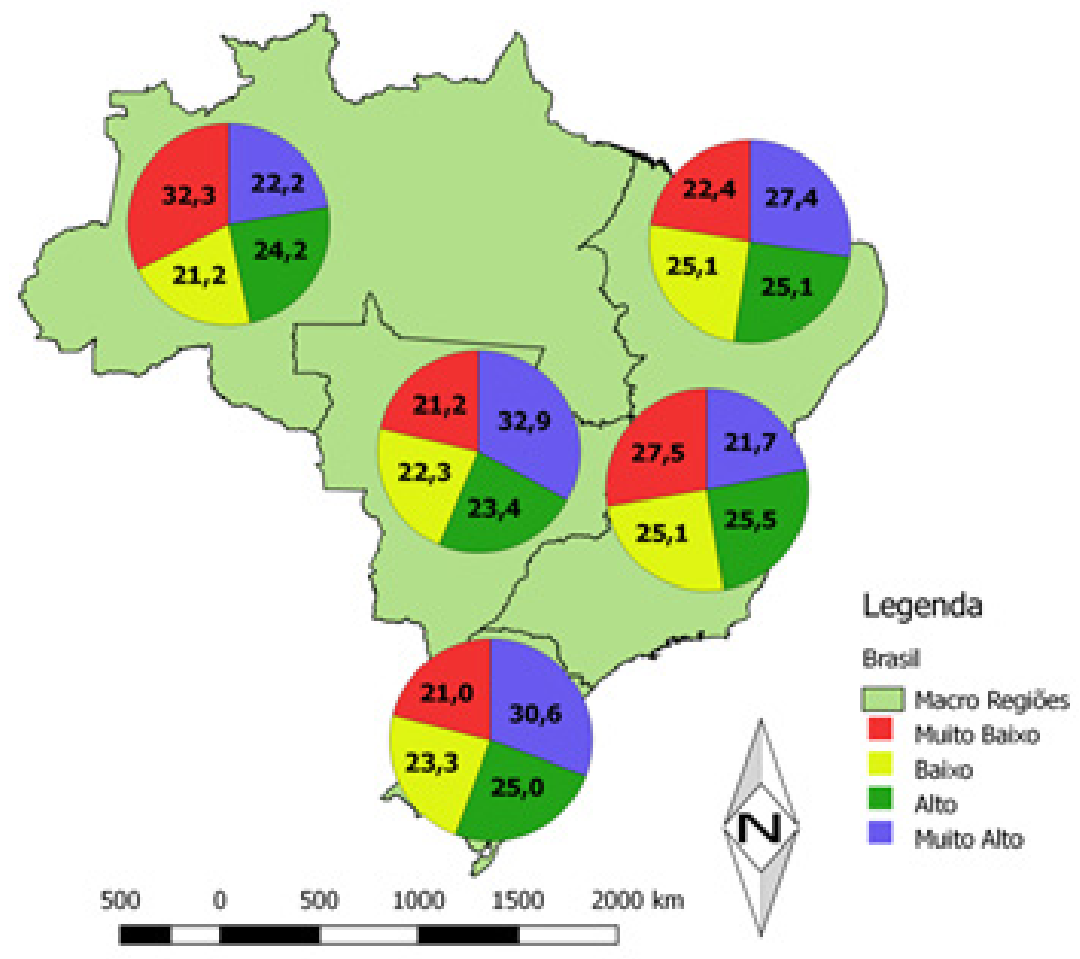

Fonte: Elaboração do autor, com base nos dados da WVS (2014).

O alto estoque de capital social da região Sul, por exemplo, pode ser explicado, em parte, pela existência de um elevado número de associações ou organizações em seu território. De acordo com dados do IBGE (s.d.) e considerando o número de habitantes das regiões brasileiras, o Sul do Brasil detinha, em 2016, quatro vezes mais associações que a região com pior nível de capital social. Ou seja, enquanto no Sul há duas associações para cada mil habitantes, no Norte havia apenas 0,5 para o mesmo número de habitantes.

\subsection{Resultados da estimação}

A Tabela 3 apresenta os resultados do modelo logit ordenado para a determinação do capital social no Brasil, considerando a razão de probabilidades das categorias de baixo capital social relativa às de alto capital social. Por meio dessa tabela, é possível analisar previamente o impacto das variáveis explicativas ${ }^{5}$ no nível de capital social, com base na razão das probabilidades, expressas pela $\exp \left(\beta_{k}\right)$ das referidas variáveis.

Iniciando a análise pela renda, escrenda $a_{i}$, constata-se que essa variável pode contribuir para um aumento no nível de capital social por um fator de 1,05 vez. Esse impacto gerado pela renda é semelhante àquele gerado pela idade, 1,03 vez. Contudo, entre todas as variáveis explicativas que foram consideradas, aquela que mais parece impactar positivamente o nível de capital social de um indivíduo é a dummy que indica se o entrevistado tem curso superior, dsup. Sua contribuição expressa por um fator de 1,93 vez é maior não somente em relação às duas variáveis acima mencionadas, como também em relação às dummies referentes às regiões

\footnotetext{
${ }^{5}$ O nível de significância das variáveis que se mostraram estatisticamente significantes variam de $1 \%(D S u p)$ a cerca de $8 \%\left(D C O_{i}\right)$, conforme pode ser observado na Tabela 3.
} 
Centro-Oeste, Sul e Nordeste, nas quais residem os entrevistados (em torno de 1,5 vez), bem como ao fato de ser católico (impacto na ordem de 1,3 vez).

Tabela 3 - Regressão logística para a probabilidade de capital social no Brasil

\begin{tabular}{|c|c|c|c|c|}
\hline \multicolumn{5}{|c|}{ Variável dependente: nível de capital social } \\
\hline Variáveis explicativas & Coef. & Des. Pad. & $\mathbf{Z}$ & Valor $-p$ \\
\hline EscRenda $_{i}$ & 0,049 & 0,028 & 1,77 & 0,077 \\
\hline Idade $_{i}$ & 0,034 & 0,018 & 1,92 & 0,055 \\
\hline $\mid d a 2_{i}$ & 0,000 & 0,0002 & $-1,39$ & 0,164 \\
\hline DMasc $_{i}$ & $-0,106$ & 0,113 & $-0,94$ & 0,348 \\
\hline DSup $_{i}$ & 0,661 & 0,186 & 3,55 & 0,000 \\
\hline DAnalf $_{i}$ & $-0,111$ & 0,132 & $-0,84$ & 0,401 \\
\hline DBran $_{i}$ & $-0,017$ & 0,125 & $-0,13$ & 0,895 \\
\hline DNegr $r_{i}$ & 0,064 & 0,175 & 0,36 & 0,716 \\
\hline DCatol $_{i}$ & 0,265 & 0,147 & 1,80 & 0,071 \\
\hline Devang & 0,187 & 0,162 & 1,15 & 0,248 \\
\hline $\mathrm{DNE}_{\mathrm{i}}$ & 0,418 & 0,215 & 1,94 & 0,052 \\
\hline $\mathrm{DCO}_{\mathrm{i}}$ & 0,464 & 0,269 & 1,73 & 0,084 \\
\hline $\mathrm{DSE}_{\mathrm{i}}$ & 0,113 & 0,210 & 0,54 & 0,589 \\
\hline DSUL & 0,455 & 0,242 & 1,88 & 0,060 \\
\hline
\end{tabular}

Fonte: Estimativa do autor, com base nos dados da WVS (2014).

Vale lembrar que, em aplicações econômicas, o modelo logit, seja ele ordenado, seja ele binário, não permite uma interpretação direta de seus coeficientes, sendo necessário para tal o cálculo de seus efeitos marginais. Sendo assim, a Tabela 4 apresenta os resultados da probabilidade da variação marginal captada pelos níveis de capital social - de um a quatro, conforme exposto na tabela -, dada a variação de cada regressor.

Isso posto, com respeito à variável escRend ${ }_{\mathrm{j}}$, os resultados indicam que níveis baixos de renda tendem a aumentar as chances de o indivíduo ter um baixo estoque de capital social, ao passo que aumentos nos rendimentos elevam, por sua vez, a probabilidade de obter maiores níveis dessa modalidade de capital. Contudo a elasticidade desse efeito se revela muito baixa. Note pela tabela que a influência da renda nos quatro níveis de capital social é inferior a 1\%. Em outros termos, o aumento em uma unidade no rendimento de um indivíduo tende a provocar, por exemplo, um aumento nos níveis de "alto e "muito alto" capital social na ordem de 0,3\% e 0,9\%.

Com relação à idade, os resultados sugerem que, à medida que os anos vão passando, as chances de um maior acúmulo de capital social aumentam. Contudo não se pode afirmar que essa evidência se mantém ou não em idades mais avançadas, dado o fato de o regressor, $1 d a z_{i}$, não ter apresentado significância estatística. Assim sendo, não é possível comprovar ou refutar a ideia de que a trajetória do estoque de capital social ao longo da vida assume a forma de " $U$ " invertido, como é usual analisar em vários estudos sobre diversos temas em que se considera, entre os regressores, a idade elevada ao quadrado.

Confirmando a análise feita com base na razão das probabilidades, Tabela 3, a variável que mais influencia o estoque de capital social parece ser a dummy dSup. Observe, por exemplo, que ter um curso superior, quando comparado aos ensinos fundamental e médio, tende a diminuir a probabilidade de um indivíduo ter um nível classificado como "muito baixo" de capital social em 
10,6\%. Considerando o outro extremo, as chances de ter um nível "muito alto" desse capital tende a aumentar em torno de $14 \%$ com o curso superior. Já no que diz respeito aos não escolarizados, $d A n a l f$, não houve significância dessa variável, do ponto de vista estatístico, com nenhum dos quatro níveis de capital social considerados.

Surpreendentemente, a variável $D C a t o I_{\mathrm{i}}$, incluída a fim de verificar se ser católico pode influir negativamente no acúmulo de capital social de um indivíduo, apresentou um resultado positivo ao se comparar com outros tipos de religião (umbanda, testemunha de jeová etc.), sugerindo que os católicos são mais propensos a ter maiores níveis de capital social. Para o nível "muito alto" de capital, por exemplo, essa propensão é na ordem de 5\%. Esse resultado encontrado para o Brasil contraria, portanto, a afirmativa de Putnam et al. (1993) de que os católicos têm, em média, um baixo nível de capital social devido a suas relações tenderem a ser pouco horizontais e mais hierarquizadas que as demais religiões. É válido ressaltar que nada se pode inferir com relação ao grupo de evangélicos, já que o regressor dEvang ${ }_{i}$ não apresentou significância estatística.

Com relação às regiões, com exceção do Sudeste, que não obteve significância estatística, todas as demais regiões indicam um impacto positivo na formação de capital social quando comparadas à região Norte. Ao considerar a penúltima coluna da Tabela 4, melhor nível de capital, residir no Centro-Oeste do Brasil pode impactar as chances de aumento de capital social em 9,5\%; para a região Sul, esse percentual é de 9,2\%; e, para o Nordeste, 8,2\%. Ao analisar os níveis de capital social por região, subitem anterior, esse resultado já era esperado, visto que a região Norte apresentou, entre todas as regiões, aquela com menor grau de capital social.

Por fim, ser do sexo masculino parece não ser um fator determinante para o acúmulo de capital social de um indivíduo, uma vez que o valor-p não apresentou nenhuma significância. O mesmo parece ocorrer com relação às dummies relacionadas à raça, $D B r a n_{i}$ e $D N e g r_{i}$, quando confrontadas com demais raças (amarelo, pardo, indígena). Isso pode levar à constatação de que parece não haver discriminação de gênero ou de raça no processo de formação de capital social de um indivíduo.

Tabela 4 - Efeitos marginais para a probabilidade de capital social no Brasil

\begin{tabular}{|c|c|c|c|c|c|c|c|c|}
\hline Variáveis & $\begin{array}{l}\mathrm{Dy} / \mathrm{dx} \\
\text { (1) }\end{array}$ & $\begin{array}{c}\text { Valor - p } \\
\text { (1) }\end{array}$ & $\begin{array}{l}\mathrm{Dy} / \mathrm{dx} \\
\text { (2) }\end{array}$ & $\begin{array}{c}\text { Valor - } p \\
\text { (2) }\end{array}$ & $\begin{array}{l}D y / d x \\
\text { (3) }\end{array}$ & $\begin{array}{c}\text { Valor - p } \\
\text { (3) }\end{array}$ & $\begin{array}{l}D y / d x \\
\text { (4) }\end{array}$ & $\begin{array}{c}\text { Valor }-p \\
\text { (4) }\end{array}$ \\
\hline EscRenda $_{i}$ & $-0,009$ & 0,078 & $-0,003$ & 0,083 & 0,003 & 0,085 & 0,009 & 0,077 \\
\hline Idade $_{i}$ & $-0,006$ & 0,055 & $-0,002$ & 0,061 & 0,002 & 0,062 & 0,006 & 0,055 \\
\hline $1 d a 2_{i}$ & 0,0000 & 0,164 & 0,0000 & 0,169 & 0,000 & 0,170 & 0,0000 & 0,164 \\
\hline DMasc $_{i}$ & 0,020 & 0,351 & 0,007 & 0,342 & $-0,007$ & 0,359 & $-0,020$ & 0,346 \\
\hline DSup $_{i}$ & $-0,106$ & 0,000 & $-0,055$ & 0,003 & 0,021 & 0,000 & 0,139 & 0,001 \\
\hline DAnalf $_{i}$ & 0,021 & 0,406 & 0,007 & 0,387 & $-0,007$ & 0,418 & $-0,021$ & 0,395 \\
\hline DBran $_{i}$ & 0,003 & 0,895 & 0,001 & 0,895 & $-0,001$ & 0,895 & $-0,003$ & 0,895 \\
\hline$D N e g r_{i}$ & $-0,012$ & 0,712 & $-0,004$ & 0,724 & 0,004 & 0,705 & 0,012 & 0,719 \\
\hline DCatol $_{i}$ & $-0,049$ & 0,072 & $-0,017$ & 0,071 & 0,017 & 0,078 & 0,050 & 0,070 \\
\hline DEvang $_{i}$ & $-0,034$ & 0,238 & $-0,013$ & 0,273 & 0,011 & 0,216 & 0,036 & 0,257 \\
\hline$D N E_{i}$ & $-0,073$ & 0,040 & $-0,030$ & 0,077 & 0,021 & 0,018 & 0,082 & 0,062 \\
\hline$D O_{i}$ & $-0,077$ & 0,052 & $-0,037$ & 0,132 & 0,019 & 0,001 & 0,095 & 0,108 \\
\hline$D S E_{i}$ & $-0,021$ & 0,587 & $-0,008$ & 0,594 & 0,007 & 0,582 & 0,022 & 0,591 \\
\hline$D S U L_{i}$ & $-0,077$ & 0,040 & $-0,035$ & 0,097 & 0,020 & 0,005 & 0,092 & 0,077 \\
\hline
\end{tabular}

Fonte: Estimativa do autor com base nos dados da WVS (2014). Para as variáveis dummies dy/dx é a mudança discreta de 0 para 1. 
Alguns dos resultados produzidos por este estudo convergem com os de Parts (2013), que, ao realizar um estudo da dinâmica do capital social para países da União Europeia e países vizinhos, observou que o nível de escolaridade, a renda e a idade influem positivamente no capital social das pessoas, sendo também a educação a que mais contribui para esse tipo de capital. É válido relembrar que a educação é também um importante determinante para a formação de capital social no estudo realizado por Downing (2011).

Especificamente com relação à influência da renda, Ribeiro e Araújo (2018), Tenzin, Otsuka e Natsuda (2013), Kimsun (2012) e Hassan e Birungi (2011) concluíram em seus estudos sobre a relação do capital social com a pobreza que esse capital pode provocar um aumento na renda, logo, por causalidade reversa, é de se esperar que o contrário também ocorra. Ou seja, melhores níveis de renda tendem a corroborar para maiores níveis de capital social, conforme se demonstrou na presente pesquisa.

Já no que tange às dummies relativas às religiões, Alexander (2007) constatou que a frequência na participação na igreja está negativamente correlacionada com os níveis de capital social nos Estados Unidos, resultado este que diverge com aquele que pôde ser observado para o Brasil.

Com base nos resultados desta pesquisa, sugere-se a intensificação de políticas sociais voltadas principalmente à educação, já que essa parece ser o principal fator determinante na formação de uma alta dotação de capital social. De fato, é de se esperar que pessoas mais escolarizadas tendam a ser mais engajadas socialmente, a fim de buscar melhores condições de vida para si e para a sociedade como um todo. Ademais, a educação pode, em média, propiciar uma sociedade integrada por pessoas com maior senso de justiça, de tolerância, de confiança e reciprocidade, atributos esses inerentes ao capital social, promovendo, assim, um maior e melhor desenvolvimento socioeconômico de uma região.

\section{CONSIDERAÇÕES FINAIS}

Este artigo analisou os fatores determinantes do capital social no Brasil. Para atender esse objetivo, calculou-se, a priori, um índice de capital social a partir da técnica de análise multivariada denominada de componentes principais. Para construção desse índice, considerou-se um total de vinte indicadores extraídos da Word Values Survey: onze deles relacionados à participação do entrevistado em organizações/associações e nove referentes à confiança interpessoal, tendo como principal base teórica o trabalho de Putnam et al. (1994). Posteriormente, estimou-se um modelo logit ordenado, seguido de seus efeitos marginais.

Os resultados revelaram que, entre todas as variáveis consideradas no estudo, aquela que mais tende a contribuir para a formação de capital social é a educação. Ter um curso superior impacta positivamente nessa modalidade de capital por um fator de 1,93 vez, ao passo que residir nas regiões Centro-Oeste, Sul ou Nordeste pode contribuir para um aumento desse capital em aproximadamente 1,5 vez. Já o impacto causado pela prática da religião católica, da renda do indivíduo e idade é de, respectivamente, 1,03, 1,05 e 1,03 vez.

Por meio dos efeitos marginais, observou-se que ter um curso superior tende a aumentar as chances de um indivíduo ter, por exemplo, uma dotação "muito alta" de capital social em 14\%. Por sua vez, quem reside no Centro-Oeste ou no Sul do Brasil está propenso a ter esse nível de dotação de capital social em 9,5\% e 9,2\%, respectivamente, e na região Nordeste, em 8,2\%. Entre aqueles entrevistados que se declararam católicos, essa propensão é de $5 \%$. Além disso, 
os resultados também sugerem que a contribuição da renda é de 0,9\%, e da idade, de 0,6\%.

Por fim, as variáveis dummies relacionadas ao gênero e à raça não apresentaram significância do ponto de vista estatístico. Desse modo, o fator discriminação, tendo como base essas variáveis, parece não interferir no processo de formação de capital social dos brasileiros.

Diante das evidências apresentadas neste estudo e considerando a relevância do capital social no processo de desenvolvimento socioeconômico e no bem-estar dos indivíduos, comprovada em diversos estudos empíricos no mundo todo, recomenda-se a intensificação de políticas públicas direcionadas fundamentalmente à expansão e à qualidade da educação no Brasil, uma vez que esse é o principal fator contribuinte para a formação de um alto nível de capital social no país.

\section{REFERÊNCIAS}

ALEXANDER, Marcus. Determinants of social capital: new evidence on religion, diversity and structural change. British Journal of Political Science, Reino Unido, v. 37, n. 2, p. 368-77, 2007.

ANDRIANI, Luca; KARYAMPAS, Dimitrios. Social capital, poverty and social exclusion in Italy. Revista Debates, Porto Alegre v. 9, n. 2, p. 177-204, jan./abr. 2015.

BEBBINGTON, Anthony; PERREAULT, Thomas. Social capital, development, and access to resources in highland Ecuador. Economic Geography, v. 75, n. 4, p. 395-418, dez. 2008.

BOURDIEU, Pierre. The forms of capital. In: Richardson J. Handbook of theory and research for the sociology of education. New York: Greenwood Press, 1986. p. 241-58.

BOURDIEU, Pierre; WACQUANT, Loïc. An Invitation to Reflexive Sociology. Chicago: University of Chicago Press, 1992.

BROOKS, Arthur. Does social capital make you generous? Social Science Quaterly, v. 86, n. 1, p. 1-15, fev. 2005.

CHRISTOFOROU, Asimina. On the determinants of social capital in Greece compared to countries of the European Union. Milão: Fondazione Eni Enrico Mattei, 2005.

COLEMAN, James Samuel. Social Capital in the Creation of Human Capital. The American Journal of Sociology, Chicago, v. 94, p. S95-S120, 1998.

DOWNING, James R. Factors influencing the variability in social capital. 2011. Tese (Doutorado em Filosofia) - University of Central Florida, Orlando, Florida, 2011.

EVANS, Peter. Government Action, Social Capital and Development: reviewing the evidence on synergy. World Development, Amsterdã, v. 24, p. 1119-32, 1996.

HASSAN, Rashid; BIRUNGI, Patrick. Social capital and poverty in Uganda. Development Southern Africa, Reino Unido, v. 28, n. 1, p. 19-37, mar. 2011.

INSTITUTO BRASILEIRO DE GEOGRAFIA E ESTATÍSTICA. Estatísticas Econômicas: Associações sem fins lucrativos no Brasil. Rio de Janeiro: IBGE, [s.d.]. Disponível em: https://www.ibge.gov.br/estatisticas/ economicas/outras-estatisticas-economicas. Acesso em: 19 jun. 2019.

IJAIYA, Mukaila A.; DAUDA, C.; PAIKO, I.; ZUBAIRU, U. Social Capital and Poverty Reduction in Nigeria: a Case Study of Minna Metropolis. International Journal of Business and Social Science, v. 3, n. 12, jun. 2012. 
INKELES, Alex. Measuring social capital and its consequences. Policy Sciences, v. 33, p. 245-68, dez. 2000.

Kimsun, Tong. Analysing Chronic Poverty in Rural Cambodia: evidence from Panel Data. Working Paper Series, n. 66, fev. 2012.

KNACK, Stephen; KEEFER, Philip. Does Social Capital have an economic payoff? A cross-country investigation. The Quaterly Journal of Economics, Oxford, v. 112, n. 4, p. 1251-88, nov. 1997.

KNACK, Stephen. Social capital, Growth and Poverty: a Survey of Cross-Country Evidence. Cap. 11. MPRA - Munich Personal RePEc Archive. World Bank, 2002.

LOCHNER, Kimberly; KAWACHI, Ichiro.; KENNEDY, Bruce. Social capital: a guide to its measurement. Health \& Place, Londres, v. 5, p. 259-70, 1999.

MANLY, Bryan. J. F. Métodos estatísticos multivariados: uma introdução. Tradução de Sara landa Carmona. 3. ed. Porto Alegre: Bookman, 2008.

MARTIN, Katie S.; ROGERS, B. L., COOK, J. T., JOSEPH, H. M. Social capital is associated with decreased risk of hunger. Social Science \& Medicine, v. 58, n. 12, p. 2645-54, 2004.

MINGOTI, Sueli Aparecida et al. Análise de dados Através de Métodos de Estatística Multivariada: uma abordagem aplicada. Belo Horizonte: UFMG, 2007.

NARAYAN, Deepa; CASSIDY, Michael F. A dimensional approach to measuring social capital: development and validation of a social capital inventory. Current Sociology, Londres, v. 49, n. 2, p. 59-102, mar. 2001.

ONYX, Jenny; BULLEN, Paul. Measuring social capital in five communities. The Journal of Applied Behavioral Science, Estados Unidos, v. 36, n. 1, p. 23-42, 2000.

PALDAM, Martin. Social capital: one or many? Definition and measurement. Journal of Economic Surveys, Dinamarca, v. 14, n. 5, p. 629-53, 2000.

PARTS, Eve. The dynamics and determinants of social capital in the European Union and Neighbouring countries. Discussions on Estonian Economic Policy: Theory and Practice of Economic Policy in the European Union, n. 1, 2013.

PEÑALOZA, Héctor Alberto Botello. Capital social y pobreza en Ecuador, 2010-2012. Ánfora, Caldas, Colombia, v. 20, n. 35, p. 93-115, 2013.

POWERS, D. A.; Xie, Y. Statistical methods for categorical data analysis. San Diego: Academic, 2000.

PUTNAM, Robert D. Bowling Alone: the collapse and revival of american community. New York: Simon and Schuster, 2000.

PUTNAM, Robert D. Tuning in, tuning out: the strange disappearence of social capital in America. Political Science and Politics, Cambridge, v. 28, n. 4, p. 664-83, dez. 1995.

PUTNAM, Robert; LEONARDI, Robert; NANETTI, Raffaella Y. Making democracy work: civic traditions in modern Italy. Nova Jérsei: Princeton University Pres. Princeton, 1994.

REGAZZI, Adair José. Análise multivariada, notas de aula INF 766. Viçosa: Departamento de Informática da Universidade Federal de Viçosa, 2000. v. 2. 
RIBEIRO, Lilian Lopes; DE ARAUJO, Jair Andrade. Capital social e pobreza no Brasil. Brazilian Journal of Political Economy/Revista de Economia Política, São Paulo, v. 38, n. 4, out./dez., 2018.

TENZIN, Galey; OTSUKA, Kozo; NATSUDA, Kaoru. Impact of Social Capital on Poverty: a Case of Rural Households in Eastern Bhutan. Ritsumeikan Center for Asia Pacific Studies (RCAPS) Working Paper Series, Japão, set. 2013.

\section{Sobre os autores:}

Lilian Lopes Ribeiro: Pós-doutora em Economia. Professora nos cursos de Economia e Finanças da Universidade Federal do Ceará (UFC), campi Sobral. Bolsista de Produtividade em Pesquisa pela Fundação Cearense de Apoio ao Desenvolvimento Científico e Tecnológico (Funcap). E-mail: liadiniz-21@hotmail.com, Orcid: http://orcid.org/0000-0001-5800-6032

Lucas Tomaz Boto: Graduado em Economia pela Universidade Federal do Ceará (UFC), campus Sobral. E-mail: lucastboto@ hotmail.com, Orcid: https://orcid.org/0000-0001-5678-1721

Fernando Daniel de Oliveira Mayorga: Doutorado em Economia pela Universidade do Arizona. Professor adjunto nos cursos de Economia e Finanças da Universidade Federal do Ceará em Sobral (UFC/Sobral). E-mail: fmayorgaufc@gmail.com, Orcid: https://orcid.org/0000-0002-8488-5234 
